\title{
Development and evaluation of herbal cream containing Curcumin from Curcuma longa
}

\begin{abstract}
Background: Curcuma longa commonly called as turmeric belongs to the family of Zingiberaceae and it is derived from the rhizomes. It is well known that curcumin has a good anti-inflammatory properties and a protective effect on the skin. Traditionally, curcumin is incorporated in many natural herbal remedies to treat skin infections and inflammation.
\end{abstract}

Purpose of the research: The purpose of this study to isolate curcumin from Curcuma longa and formulate curcumin containing moisturizing conditioning cream.

Methods: All solvents and chemical were of analytical and HPLC grade. Conventional extraction using Reflux Condensing Unit was used to isolate curcumin and then analysis was performed by by UV, TLC, and HPLC methods. By using primary emulsion method, the herbal cream was formulated. The curcumin cream was evaluated for different physiochemical tests like appearance, homogeneity, grittiness, spreadability, viscosity, drug content, determination of $\mathrm{pH}$, and test for microbial growth.

Results: Curcumin (95\%) was isolated from Curcuma longa collected from Barka Al Jabar farm, Oman. Primary emulsion method was used for the preparation of the herbal cream to ensure stability and prevent incompatibility. The results of the evaluation of different physiochemical properties were found good and matching with the standard values.

Conclusion: Curcumin was isolated from Curcuma longa. The herbal cream was formulated and evaluated by several standard methods. Evaluation results were found satisfactory and matching with the international standard values.
Volume 8 Issue 5 - 2020

\author{
Melak Mohammed Al-Busaid,' Md. Sohail \\ Akhtar,' Tanveer Alam, ${ }^{2}$ Wegdan Aly Shehata' \\ 'School of Pharmacy, College of Pharmacy and Nursing, \\ University of Nizwa, Sultanate of Oman \\ ${ }^{2}$ Natural and Medical Sciences Research Center, University of \\ Nizwa, Sultanate of Oman
}

Correspondence: Md. Sohail Akhtar, University of Nizwa, School of Pharmacy, College of Pharmacy \& Nursing, P.O. Box 33, Post Code 616 Birkat Al Mouz, Nizwa, Sultanate of Oman, Tel +968-25-446-423,

Email sohal@unizwa.edu.om, sohail276@gmail.com

Received: August 16, 2020 | Published: September 14, 2020

Keywords: Curcuma longa, Zingiberaceae, cream, Curcumin, turmeric

\begin{abstract}
Abbreviations: UV, Ultraviolet; TLC, Thin-layer chromatography; HPLC, High-performance liquid chromatography; EDC, ethylene dichloride; OD, optical density; EDTA, Ethylenediaminetetraacetic acid; q.s., quantity sufficient; Rf, retention factor
\end{abstract}

\section{Introduction}

This study was conducted to extract, purify, and isolate Curcumin from the plant Curcuma longa and then to formulate a curcumin containing herbal moisturizing cream. Curcuma longa, is a member of Zingiberaceae family; turmeric is derived from their rhizomes. The dark orange color of curcuma is due to the presence of lipophilic, polyphenolic carotenoids known as curcuminoids. ${ }^{1}$ Curcumin is known to have medicinal effects on a different types of diseases in human, and has shown the anti-proliferative effect in multiple cancers. ${ }^{2}$ It is therapeutically used in many diseases like amenorrhea, anemia, asthma, dislocation of joints, diabetes, diarrhea, cough, dyspepsia, liver disorder, loss of appetite, cough, bronchitis, hepatitis, ring worm infection, menstrual disorder, tooth ache, urinary infection, skin diseases, scorpion sting, ring worm infection, and weakness of eye sight. ${ }^{3}$ Curcuma longa usually cultivated in Cambodia, India, South China, Indonesia, Madagascar, Malaysia, the Philippines, and Viet Nam. Normally Turmeric found at the altitude of 500-900 $\mathrm{m}$ in Thailand. ${ }^{4}$

The main purpose of this study was to isolate curcumin from the Curcuma longa plant and formulate herbal cream containing curcumin to emphasize the importance of involving natural ingredients in pharmaceutical products instead of synthetic ingredients. The significance of this study can be clearly seen due to the fact that now a days there are lots of creams available in the market with synthetic chemical ingredients which can cause harmful effects on the skin. Natural ingredient based creams have very less side effects. Especially curcumin isolated from Curcuma longa that has very good effects on the skin. In addition it was reported that Curcumin has a good antiinflammatory, anti-oxidant, and anti-microbial activity and this opens a way for researchers to incorporate this to treat skin diseases. ${ }^{5-7}$

\section{Material and methods}

\section{Research duration}

This research was started in September/2018 and ended in March/2019. It was performed in School of Pharmacy Research laboratory, University of Nizwa, Sultanate of Oman.

\section{Plant and chemicals procurement}

Curcuma longa rhizomes used in this study were collected from Barka Al Jabar farm, Oman. Rhizomes were washed with running water to remove dust particles and air dried under shed. The moisture content of dried rhizome was $0.01 \pm 0.04 \mathrm{~g}$ water $/ \mathrm{g}$. Dried rhizomes were grinded to coarse powder and ready for extraction process.

\section{Conventional extraction using reflux condensing Unit}

The rhizomes of turmeric were dried in shed. Dried rhizomes were grinded into coarse powder through multi mill. 300 gm ground 
turmeric powder was weighed and loaded in the reflux condensing unit which was filled with ethylene dichloride (EDC) as extracting solvent. The extraction of the turmeric rhizome powder was carried out at $60^{\circ} \mathrm{C}$ for 4 hours. After the extraction process, the material was filtered out by Buchner funnel. Filtrate was distilled out using Yamato Rotary evaporator model RE810 under vacuum at $50^{\circ} \mathrm{C}$ to get the residue (oleoresin).

\section{Isolation of Curcumin}

Curcumin were isolated from Curcuma longa dried rhizomes powder. $^{8}$

\section{Process flow chart of Curcumin isolation}

Coarsely crushed grinded Powder

$$
\downarrow \text { Extraction }
$$

Extracted with Ethylene dichloride (EDC) for 4 hours in extraction unit at $50^{\circ} \mathrm{C}-60^{\circ} \mathrm{C}$

Filtration

Filtered the extract through Buchner funnel \& collected in a beaker for concentration

\section{Concentration}

Concentrated under vacuum at $50^{\circ} \mathrm{C}-60^{\circ} \mathrm{C}$ up to $40 \%$ of raw herb Crystallization

Collected in a beaker and left for 24 hours for crystallization Filtration

Filtered the crystal through a centrifuge

Purification

Refluxed the filtered crystal with $50 \%$ Ethyl acetate \& water for half an hour at $60^{\circ} \mathrm{C} \&$ collected in a beaker and allow cooling at $5^{\circ} \mathrm{C}$ for 4 hours

Filtration \& Washing

Separated the precipitate of curcumin through a centrifuge

$$
\text { Drying }
$$

Collected curcumin \& dried under vacuum/oven

$$
\text { Grinding }
$$

Grinded the dried curcumin through a grinder

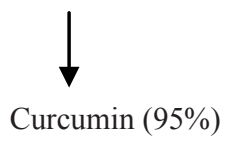

The extraction yield of Curcuma longa rhizome was then determined by using following formula.

$$
\text { Curcumin Yield }(\%)=\frac{\text { Weight of isolated Curcumin }}{\text { Weight of dried powder }} \times 100
$$

\section{Analysis of isolated Curcumin by TLC Method}

\section{Sample preparation}

One mg of curcumin was measured in electronic balance and transferred to a $10 \mathrm{ml}$ volumetric flask. A $5 \mathrm{ml}$ of ethyl alcohol was added and sonicated for 3 minutes and volume was made up to $10 \mathrm{ml}$ with ethyl alcohol.

\section{TLC Plate}

Pre-coated silica gel plates (Catalogue No.1.05554) was used.

\section{Application}

$10 \mu 1$ sample preparation mentioned above was applied using TLC applicator (Linomat IV, Camag).

\section{Solvent system}

Chloroform: Ethyl alcohol: Acetic acid

$$
95: 5 \quad: \quad 1
$$

\section{Development of TLC plate}

The TLC plate was developed in twin trough developing saturated chamber (Camag).

\section{Analysis of isolated Curcumin by UV method 90 Preparation of samples}

a. The appropriate amount of material was put into $200 \mathrm{ml}$ volumetric flask and recorded the weight, dissolved in HPLC grade ethanol, and made up the volume.

b. $1.0 \mathrm{ml}$ solution transferred into $100 \mathrm{ml}$ volumetric flask and made up the volume.

c. Stoppered the flask and mixed thoroughly by inverting it several times and then recorded the UV Absorption range (OD) at absorbance of $425 \mathrm{~nm}$.

\section{Calculation}

Calculated the percentage of total purity of curcumin

$$
\text { Total Curcumin } \%=\frac{\mathrm{OD} \times 200}{\mathrm{~W} \times 1607} \times 100
$$

Where OD $=$ absorbance of sample

$\mathrm{W}=$ weight of sample $(\mathrm{g})$

$1607=$ specific absorbance of the curcumin Standard in ethanol at $425 \mathrm{~nm}$.

\section{Analysis of isolated Curcumin by HPLC method}

\section{Chromatographic conditions}

Mobile phase

$$
\begin{aligned}
& \text { :A filtered and degassed mixture of } 35 \\
& \text { volumes of tetra hydro furan and } 65 \\
& \text { volumes of buffer. }
\end{aligned}
$$

Buffer $: 1 \%$ citric acid solution is adjusted to $\mathrm{pH} 3.0$ with ammonia solution.

Column :Silica CN, 5 size, 250 x 4.6mm 


$\begin{array}{ll}\text { Detector } & : U V \text { detector } \\ \text { Wave length } & : 424 \mathrm{~nm} \\ \text { Flow rate } & : 1.2 \mathrm{ml} / \text { minute } \\ \text { Injection volume } & : 20 \mu 1 \\ \text { Run time } & : 45 \text { Minutes. }\end{array}$

\section{Herbal Cream Preparation}

The ingredients used in herbal formulation are listed in Table 1. Propylene gycol: glycerine: sorbitol (2:1:1) is used as moisturizer conditioner.

Aqueous Phase: All water soluble ingredients dissolved in water.

Oil Phase: All ingredients which were oil soluble mixed at $75^{\circ} \mathrm{C} \pm 5^{\circ} \mathrm{C}$ in separate beakers.

The aqueous phase then poured into oil phase slowly with vigorous stirring and finally the mixture was passed through homogenizer for 30 minutes. ${ }^{11}$

Table I List of ingredients of formulation"

\begin{tabular}{lll}
\hline S. No. & Ingredients & Quantity (\%w/w) \\
\hline 1 & Curcumin (95\%) & I \\
2 & Stearic acid & 20 \\
3 & Triethanolamine & 3 \\
4 & Liquid paraffin & 7 \\
5 & Glycerine (moisturizer & 20 \\
6 & conditioner) & 4 \\
7 & Cetyl alcohol & 0.04 \\
8 & Propyl paraben & 0.2 \\
9 & Sodium metabisulphite & 0.2 \\
10 & Ethylenediaminetetraacetic acid & \\
\hline
\end{tabular}

\section{Evaluation of creams}

The prepared curcumin cream was observed visually and homogeneity, grittiness, viscosity, spread ability, $\mathrm{pH}$, and its stability studies were recorded.

\section{Viscosity}

Using a Brookfield viscometer, the viscosity of the formulated herbal cream was measured by pouring the herbal cream into the viscometer adaptor and then observed the angular velocity that was 0.5 and then increased to reach $20 \mathrm{rpm}$.

\section{Test for microbial growth in formulated cream}

The prepared herbal cream was tested for any microbial growth by streak plate method by inoculating the formulated herbal cream and the control which didn't contain the formulated cream in agar media plates. After that the plates were incubated in the incubator for 24 hours with a temperature of $37^{\circ} \mathrm{C}$ and then it was examined and compared with the control to observe any microbial growth. ${ }^{11}$

\section{Homogeneity \& Appearance}

After the cream has been set in the container, the formulation was tested for homogeneity by visual appearance and by physical touch. The appearance was determined by examining the pearlscence, the roughness, and the color. ${ }^{12}$

\section{Grittiness}

Formulation was evaluated with the help of compound microscope to observe for the presence of any particles. ${ }^{13}$

\section{Determination of $\mathrm{pH}$}

A calibration by a standard buffer solution was done to the $\mathrm{pH}$ meter and then $0.5 \mathrm{~g}$ of the formulated herbal cream was taken and mixed properly with $50 \mathrm{ml}$ distilled water. Then, the $\mathrm{pH}$ of the cream was determined by the $\mathrm{pH}$ meter at room temperature. ${ }^{14}$

\section{Spreadability}

Spread ability is measured by time in seconds utilized by two glass slides to slip off from cream, lesser time taken for separation of two slides, denotes the better the spreadability. Measuring the spreadability was done by adding $3 \mathrm{~g}$ of the herbal cream between two slides and pressed it to get a thin layer which is uniform and then a $1000 \mathrm{~g}$ weight was placed for 5 minutes. Using a pan, $10 \mathrm{~g}$ of weight was added to it. The upper plate was attached to a string which is also attached to a hook so that the plate can be pulled. The time taken for the upper plate to go over the lower one to cover $10 \mathrm{~cm}$ of distance was recorded. After that, the spreadability was calculated using the following formula. ${ }^{15}$

$$
\mathrm{S}=\frac{\mathrm{M} \times \mathrm{L}}{\mathrm{T}}
$$

Where $\mathrm{M}=$ weight tied to upper slide, $\mathrm{L}=$ length of glass slides, $\mathrm{T}$ $=$ time taken to separate the slides

\section{Drug content}

To judge the uniformity of prepared cream, a UV-Visible spectrophotometer was used. About $2 \mathrm{~g}$ of the formulated herbal cream was dissolved and mixed with $100 \mathrm{ml}$ of the phosphate buffer of a $\mathrm{pH}$ 7.4. Then, this solution was filtered using a filter paper and then it was analyzed at a wavelength of $254 \mathrm{~nm}$ against a blank that consist of buffer of a $\mathrm{pH} 7.4 .^{16}$

\section{Stability study}

Stability study over a period of three months was conducted. The physical appearance, $\mathrm{pH}$ value, drug content, were determined periodically after the $1^{\text {st }}, 2^{\text {nd }}$ and $3^{\text {rd }}$ month after cream preparations. The stability of the formulated herbal cream was tested under different temperature which are $2^{\circ} \mathrm{C}, 25^{\circ} \mathrm{C}$ and $37^{\circ} \mathrm{C} .{ }^{17}$

\section{Results}

Around $300 \mathrm{gm}$ of turmeric is used to isolate the curcumin (95\%) and $9.13 \mathrm{gm}$ of curcumin was obtained. The percentage yield of curcumin was $3.04 \%$. The moisturizing conditioner cream was formulated by incorporating curcumin with the wet gum method.

\section{Analysis of isolated Curcumin by UV method}

Purity of isolated curcumin was measured by UV method and was $95.287 \%$ 


\section{Development of TLC plate}

The TLC plate was developed in twin trough developing saturated chamber (Camag). Three spots were detected both for sample and standard curcumin.

\section{Analysis of isolated Curcumin by HPLC method}

Isolated curcumin was also analyzed by HPLC method and the peaks of curcumin, Demethoxy curcumin and Bis-demethoxy curcumin was detected.

\section{Evaluation of creams}

The appearance of the formulated herbal cream was judged by visual observations. The color of the cream was found intense bright yellow. Results revealed that the formulated herbal cream had a very smooth texture. Formulation showed spreadability. The $\mathrm{pH}$ of the formulated cream was found to be 4.5 at room temperature. The formulated herbal cream was tested for the presence of microorganisms by culturing it with Muller Hinton agar medium. There was no sign of pathogenic microbial growth after incubation for 24 hours at $37^{\circ} \mathrm{C}$ and it was comparable with the control. The result of stability studies showed that the cream remained stable after stability studies.

\section{Discussion}

Curcumin was isolated from Curcuma longa and the percentage yield was satisfactory. For measuring identity and purity there were three methods used like TLC, HPLC and UV. After observing TLC plate it was clear that both sample and isolated curcumin have same Rf value (Figure 1). HPLC data also supported the presence of curcumin (Figure 2). UV method used in this experiment showed purity of the isolated compound which is of $95.287 \%$ and is matching with the standard value of purity. Curcumin containing cream was formulated and the color was bright yellow and had a cosmetically appealing appearance and it was homogenous with no indication of two phase formation. Formulated cream was easily spreadable, with fair mechanical properties and acceptable bio adhesion. The $\mathrm{pH}$ of the skin normally ranges from 4 to 6 and $\mathrm{pH}$ of the formulated cream was found to be 4.5 at room temperature and was similar to the skin's normal $\mathrm{pH}$ value. Stability studies results showed that there were no significant changes in the $\mathrm{pH}$ value, physical appearance and drug content of the cream, after storing at different temperature conditions for three months.

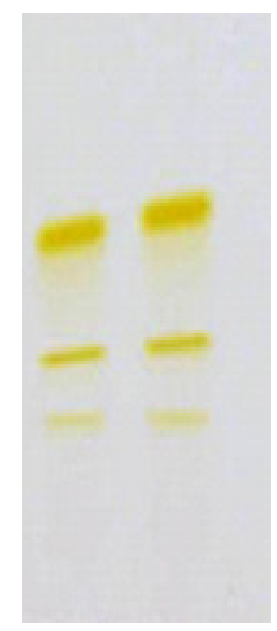

Isolated Std.

Figure I TLC plate of Curcumin.
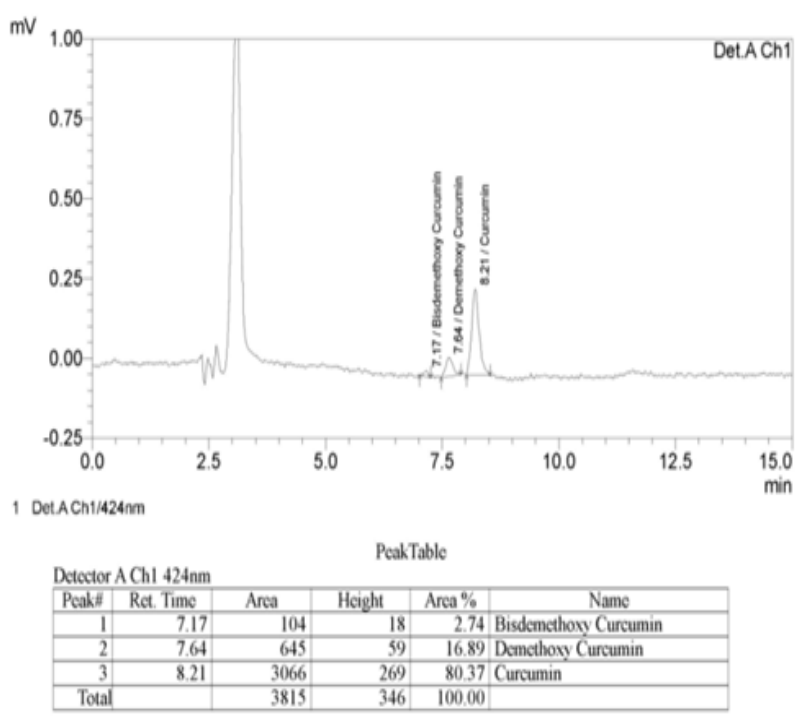

Figure 2 HPLC data of Curcumin.

\section{Conclusion}

The purpose of this study was to develop a herbal cream. The formulated Curcumin containing moisturizing conditioning cream was evaluated for several physiochemical tests and the results were found according to the standard value. Curcumin is a natural pigment obtained from Curcuma longa with considered medicinal values. This herbal cream is one of the good alternatives in place of synthetic cream. Further detailed stability studies are needed to improve the overall quality of the products.

\section{Acknowledgments}

Thanks for the University of Nizwa, Sultanate of Oman for providing facilities to do this research work.

\section{Conflicts of interest}

Authors has no conflict of interest to disclose.

\section{References}

1. Samarasinghe K, Wenk C, Silva KF, et al. Turmeric (Curcuma longa) root powder and mannanoligosaccharides as alternatives to antibiotics in broiler chicken diets. Asian-Australas J Anim Sci. 2003;16(10):1495-500.

2. Hayakawa H, Minaniya Y, Ito K, et al. Difference of curcumin content in Curcuma longa L. (Zingiberaceae) caused by hybridization with other Curcuma species. Am J Plant Sci. 2011;2(2):111-119.

3. Akram M, Shahab-Uddin AA, Usmanghani KH, et al. Curcuma longa and curcumin: a review article. Rom J Biol Plant Biol. 2010;55(2):65-70.

4. Sirirugsa P, Larsen K, Maknoi C. The genus Curcuma L. (Zingiberaceae): distribution and classification with reference to species diversity in Thailand. Gard Bull (Singap). 2007;59(1\&2):203-220.

5. Araujo CA, Leon LL. Biological activities of Curcuma longa L. Mem Inst Oswaldo Cruz. 2001;96(5):723-728.

6. Jurenka JS. Anti-inflammatory properties of curcumin, a major constituent of Curcuma longa: a review of preclinical and clinical research. Altern Med Rev. 2009;14(2):141-153.

7. Alavijeh PK, Alavijeh PK, Sharma D. A study of antimicrobial activity of few medicinal herbs. Asian J Plant Sci Res. 2012;2(4):496-502. 
8. Shettar R, Gaika VG, Srivastava, et al. Process recovery of pure curcumin from Turmeric rhizomes. The Godavari sugar mills limited. Indian Patents. 205792:42/2008.

9. European Food Safety Authority. Refined exposure assessment for curcumin (E 100). EFSA Journal. 2014;12(10):1-43.

10. Stankovic I. Curcumin: Chemical and Technical Assessment (CTA). JECFA. 2004;(8):1-8

11. Nair SS, Mathew M, Sreena K. Formulation and evaluation of herbal cream containing Curcuma longa. Int J Pharm Chem Sci. 2012;1(4):1362-1368.

12. Kaur LP, Garg R, Gupta GD. Development and evaluation of topical gel of minoxidil from different polymer bases in application of alopecia. Int $J$ Pharm Pharm Sci. 2010;2(3):43-47.
13. Tarun J, Susan J, Suria J, et al. Evaluation of $\mathrm{pH}$ of bathing soaps and shampoos for skin and hair care. Indian J Dermatol. 2014;59(5):442-444.

14. Aswal A, Kalra M, Rout A. Preparation and evaluation of polyherbal cosmetic cream. Der Pharmacia Lettre. 2013;5(1):83-88.

15. Dhase AS, Khadbadi SS, Saboo SS. Formulation and evaluation of vanishing herbal cream of crude drugs. AJ Ethno. 2014;1(5):313-318.

16. Ubaid M, Ilyas S, Mir S, et al. Formulation and in vitro evaluation of carbopol 934-based modified clotrimazole gel for topical application. An Acad Bras Ciênc. 2016;88(4):2303-2317.

17. Lokes MS, Gurunath KP, Chandrasekar SB, et al. Formulation and evaluation of herbal formulations (Ointment, Cream, Gel) containing Tridax procumbens and Areca catachu. IJSRIS. 2017;6(3):97-100. 